\title{
The End-to-End Rate Control in Multiple-Hop Wireless Networks: Cross-Layer Formulation and Optimal Allocation
}

\author{
Chengnian Long, Member, IEEE, Bo Li, Senior Member, IEEE, Qian Zhang, Senior Member, IEEE, Bin Zhao, \\ Bo Yang, Xinping Guan, Senior Member, IEEE
}

\begin{abstract}
In this paper, we study the theoretical problem of the end-to-end rate assignment for multi-hop wireless networks. Specifically, we consider the problem of joint congestion control, random access and power control design with multihop transmissions and interference-limited link rates. In order to address both the end-to-end throughput maximization and energy efficiency, we formulate this problem into a cross-layer design problem under a realistic interference-based communication model, which captures the attainable link capacity in practice. There are primarily three challenges in this design: 1) how to formulate the cross-layer design; 2) how to solve the nonconvex and non-separable problem efficiently; more importantly 3) under a reasonably complexity, how to design a distributed algorithm that can realize this formulation while maintaining the architectural modularity among different layers.

First, we propose a novel method that can convert a nonconvex and non-separable programming into an equivalent convex programming problem. The problem is solved by a dual decomposition technique. We show that the resulting algorithm can be practically realized. We then design a distributed algorithm that jointly considers random access and power control to adapt for the transport layer congestion status. Simulation results confirm that the proposed algorithm can achieve close to the global optimum within reasonable convergence times.
\end{abstract}

Index Terms-Multi-hop wireless networks, cross-layer design, power control, random access, congestion control, interferencebased communication model, geometric programming.

\section{INTRODUCTION}

$\mathbf{T}$ HIS demand for efficient and fair allocation of wireless resource increases with the rapid development and deployment of wireless multi-hop network in various fields, such as sensor networks and mobile control systems. One of the

Manuscript received May 14, 2007; revised November 12, 2007. The research was supported in part by grants from RGC under the contracts 616406,616207 , and 622407 , by grants from NSFC/RGC under the contract N_HKUST603/07 and N_HKUST609/07, by grants from HKUST under the contract RPC06/07.EG 27 and RPC06/07.EG05, by a grant from National Basic Research Program of China (973 Program) under the contract 2006CB303100, by a grant from Nokia Research under the contract NOKIA001-BL.06/07, by grants from NSF China under the contracts 60629203, 60429202, 60573115, 60525303, 60404022, and 60604012, and NSF of Hebei Province under the contracts F2006000270, and F2005000390.

C. N. Long, B. Zhao, and B. Yang are with the Centre for Networking Control and Bioinformatics (CNCB), Department of Electrical Engineering, Yanshan University, Qinhuangdao, 066004, Hebei Province, P.R. China (email:dylcn@ysu.edu.cn).

B. Li and Q. Zhang are with Department of Computer Science and Engineering, Hong Kong University of Science and Technology, Clear Water Bay, Kowloon, Hong Kong, China (e-mail:\{bli, qianzh\}@cse.ust.hk).

X. P. Guan is with Department of Automation, School of Electronic, Information, and Electrical Engineering, Shanghai Jiaotong University, Shanghai, P. R. China 200240 (e-mail:xpguan@sjtu.edu.cn).

Digital Object Identifier 10.1109/JSAC.2008.080513. key issues is to develop distributed control mechanisms that can rapidly respond to the changing network conditions while maintaining the system efficiency and fairness simultaneously. From control theory, feedback is one of the most efficient ways in achieving system stability and robustness under uncertain, time-varying and information-limited environments. In wired networks, Internet congestion control, such as TCP/AQM, is a representative distributed control mechanism in the form of feedback-based dynamic response to network congestion. As shown in Fig. 1 [16], the network is modeled as a collection of individual users and network components that are in constant negotiation with one another. The main innovation from this line of work (e.g., [8], [14], [15], [16]) is to formulate an optimization-based framework which provides an interpretation of various congestion control mechanisms and forwardengineering protocol design. Although congestion control has been studied extensively for wired networks, these results cannot be applied directly to multi-hop wireless networks. In wired networks, the network capacity region (i.e., the set of feasible data rates) is of a simple form. That is to say, the sum of the data rates at each link should be less than the link capacity, which is usually known and fixed. In multi-hop wireless networks, the capacity of each radio link depends on the signal and interference levels, which is further complicated by the power allocation and transmission schedule at vicinity links. Hence, the capacity region is usually of a complex form that critically depends on the way where resources at the underlying physical and MAC layers are utilized. This becomes particularly challenging when designing an end-toend rate control in wireless multi-hop networks.

One possible way is to use the traditional layered approach (see, e.g. [25], [26]). As shown in Fig. 2, this is usually an open loop system for the whole protocol stack. Herein, the power control and MAC are designed based on some local criteria. With the prior knowledge of the fixed capacity region $f$, the rate control at transport layer is similar with the Internet rate control algorithms. This approach essentially attempts to make rate control oblivious of the dynamics of the underlying layers. Due to the lack of the end-to-end consideration at the lower stack of protocols, the power control and MAC cannot adapt to the end-to-end service requirements. Thus, such a layered approach often results in a conservative rate allocation and energy wastage. In this paper, we study a distributed rate control problem in a multi-hop wireless network with interference-limited link rates and utilizing the cross-layer design philosophy. Our focus is on how to construct closed- 


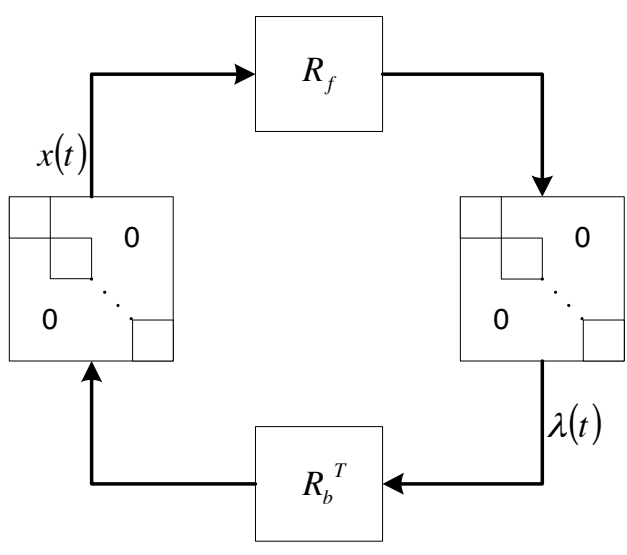

Fig. 1. General feedback structure for Internet congestion control system (a copy from Figure 1, [16]).

loop power control and MAC in the form of congestion feedback information.

\section{A. Related Work and Motivating Example}

It has been widely recognized that cross-layer design can potentially lead to substantial performance gains in wireless networks (see, e.g. [6], [9], [11], [18], [20], [24]). The efficiency and fairness of resource allocation are of great importance in multi-hop wireless networks. This section summarizes the relevant works on jointly optimal design of rate control, MAC and power control.

1) Joint Rate Control and MAC: The joint rate control and scheduling-based MAC design was studied extensively (see, e.g. [2], [3], [11], [12], and [20]). In [20], Price and Javidi investigated the distributed rate assignments problem in CDMA-based wireless networks. They presented a modular implementation of a distributed rate assignment algorithm at the MAC and transport layer, which regulated rates separately based on the congestion and interference constraints. Lin et al. discussed the joint problem of allocating data rates and stabilizing scheduling policy in a multi-hop wireless network [11]. In [11], the scheduling policy in the crosslayer congestion control scheme requires solving a global optimization problem in each iteration, which can be complex. An imperfect scheduling scheme was proposed in [12], which designed a suboptimal solution for the scheduling problem with significantly less computation overhead. In [3], Chen et al. addressed the joint congestion control and media access control for ad hoc wireless networks, where the rate allocation is formulated as a utility maximization problem with the constraints that arise from contention for channel access. Furthermore, their model is reformulated in the consideration of the routing and the network with time-varying channel, multirate devices [4]. In [2], an asynchronous congestion control model was proposed to account for the effect of the information-exchange delay. It showed that the proposed joint asynchronous congestion control and scheduling algorithm supported at least one-third of the throughput supportable by any algorithm including centralized algorithms. Compared to schedule-based MAC scheme, random access scheme incurs less computation and implementation overhead, which

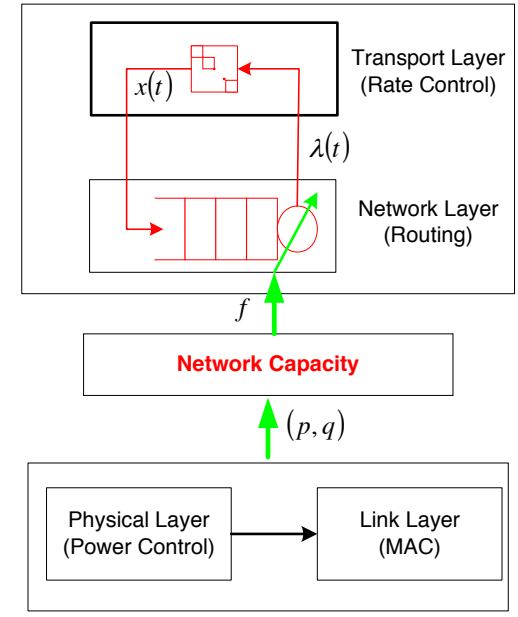

Fig. 2. The open loop control structure for layered protocol stack in wireless system.

is widely applied. Therefore, it is important to study the rate control algorithm design under random access protocols. Recently, Wang and Kar addressed the end-to-end proportionally fair rate control problem in a multi-hop random access network with a general network topology [23]. Two crosslayer algorithms were proposed to solve the problem, both of which can be implemented in a distributed manner. The stochastic noisy feedback, however, is practically inevitable in the distributed algorithm implementation for the cross-layer design, especially for the wireless multi-hop networks with random access manner. In [27], Zhang et al. made use of combination of advanced tools in stochastic approximation, martingale theory and convex analysis to study the impact of stochastic noisy feedback on the general distributed algorithms based on different decomposition methods.

2) Joint Rate Control and Power Control: A jointly optimal congestion control and power-control algorithm for a general ad hoc network was presented in [5] to enhance the overall network performance. The main contribution was the proposal of a framework on how physical layer resource allocation can be adapted to enhance the end-to-end utilities.

A distinctive character of wireless networks is that the attainable link capacity is determined by both link scheduling and power level decision. However, there is little work on jointly optimal design of rate control, random access and power control due to the difficulty in solving non-convex, non-separable programming problem. Furthermore, most of MAC designs adopt a collision-based communication model (node exclusive collision model [2] and $K$-hop collision model [21]) for capturing the link access and network capacity, which often ignore the power influence from neighborhood nodes. These models imply that simultaneous transmissions on proximate links necessarily collide with each other, which is not necessarily true in a signal to interference plus noise ratio (SINR) environment. In fact, the mutual interference links can be activated synchronously if the transmission powers of the different senders are properly adjusted [17]. To illustrate the difference between collision-based and interference-based communication model, we present a simple motivating example as following. The network topology is shown in Fig. 3 . 


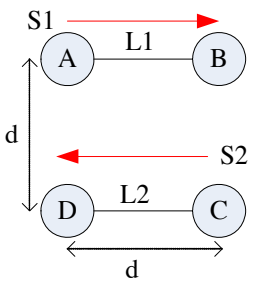

Fig. 3. A simple network topology for motivation example.

With the collision-based model, the attainable link capacity is expressed as

$$
f_{l}=c_{l} q_{l}\left(1-\Im_{r_{l}}\right) \Pi_{v \in N_{t o}^{I}(l)}\left(1-\Im_{v}\right)
$$

where $c_{l}=\log \left(1+\frac{h_{t_{l} r_{l}} p_{l}^{\max }}{\sigma^{2}}\right)$ is the maximum link capacity [23]. Assume the two users' utility functions are $U(x)=$ $\log x$. Fig. 4 depicts the attainable link capacity with different $p^{\max }$ under collision-based and interference-based communication model (2)-(4), where $f 1=0.25 \log \left(1+\frac{h p^{\max }}{\sigma^{2}}\right)$ is the attainable link capacity in theory with collision-based model, $f 2=0.5 \log \left(1+\frac{h p^{\max }}{\sigma^{2}+\frac{1}{2 B} h p^{\max }}\right)$ is practical attainable link capacity with $p_{1}=p_{2}=p^{\max }, q_{1}=q_{2}=0.5$. Given $p_{1}=$ $p_{2}=0.5 p^{\max }, q_{1}=q_{2}=1, f 3=\log \left(1+\frac{0.5 h p^{\max }}{\sigma^{2}+\frac{1}{2 B} h p^{\max }}\right)$ is attainable link capacity with the the interference-based communication model. $f 4$ is the optimization solution for the convex programming problem (9) in Section II. Thus, we have the following observations from the illustrative example.

1) The interference-based communication model (2)-(4) is corresponding to the attainable link capacity in practice, which may enhance the end-to-end throughput utility with the same energy consumption from $f 2$ and $f 3$.

2) It is profound importance of choosing a proper transmitting power in the interferenc-limited environment. Increasing the power level may lead to high interference, which results in the total throughput utility reducing and energy wastage.

The above observations motivate us to jointly consider the design of rate control, interference-based random access and power control. One of the key issues is how to characterize the influences from resource allocations in MAC layer and physical layer on the end-to-end throughput.

\section{B. Summary of Results}

In this paper, we adopt an interference-based communication model with the notion of SINR for the link capacity. That is to say, a message is successfully received by a node if the average SINR exceeds a certain hardware-specific threshold. We model the link capacity with Shannon mean for SINR, which is a function of power level and link access probability. Therefore the interference-based communication model can more accurately capture the attainable network capacity in practice. Moreover, compared to scheduling-based MAC schemes, the random access scheme is more flexible and easy to implement in a distributed manner. Thus, from practical engineering point of view, the important problem is the joint design of random access and power control for

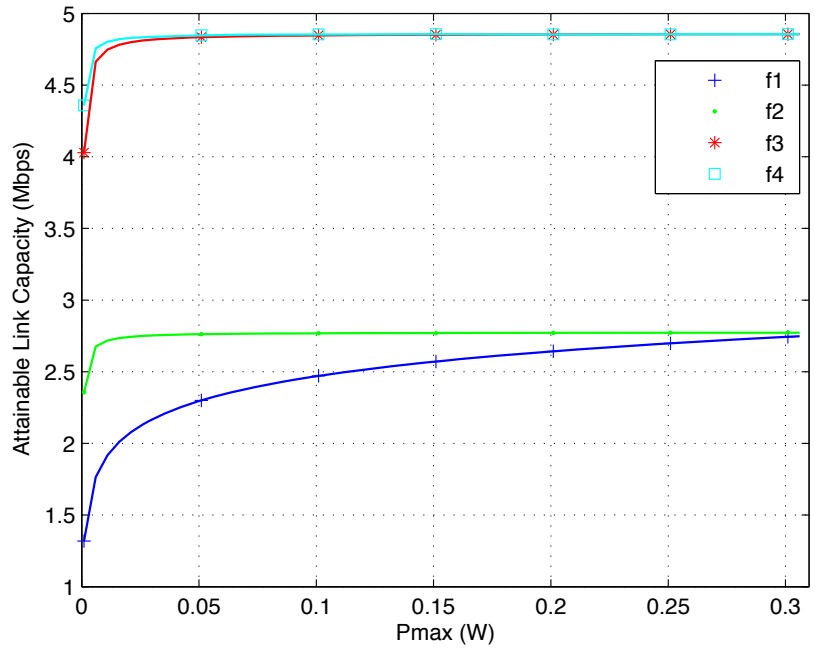

Fig. 4. The attainable link capacity comparion between collision-based and interference-based communication model.

adapting to transport layer; further, how to implement this in a distributed manner and enhance the end-to-end utilities. Our study is motivated by solving these critical problems. The main contributions are summarized below:

- We propose a framework for joint rate control, random access and power control underlying the interferencebased communication model.

- We propose a method combining geometric programming with variable substitute, which converts a nonconvex, non-separable nonlinear programming into convex programming problem. The proposed method falls into the "layering as optimization decomposition" approach. Thus, the three-layer joint optimization design can maintain the architectural modularity among different layers.

- We propose a distributed random access and power control algorithm to perform the resource allocation in according with the congestion prices. We show that both the end-to-end throughput utility and energy efficiency can be significantly improved with the simultaneous optimization of three layers.

The rest of the paper is organized as follow. Section II introduces the system model we consider in this paper. Section III presents an equivalent convex formulation for the end-to-end rate control problem. With the dual decomposition technique, the cross-layer design is separated as rate control subproblem and joint random access and power control subproblem. The protocol implementation issues are discussed in Section IV. We present the Jointly Optimal Congestion-Control, RandomAccess and Power-Control (JOCRP) Algorithm, which can be implemented in a distributed manner with message passing. Section $\mathrm{V}$ conducts the simulation investigate to support our analytical observations. Finally, conclusions are made in Section VI.

\section{System Model and Problem Formulation}

A multi-hop wireless network can be represented as a directed graph $G=(V, L)$, where $V$ is the set of nodes in 
the network and $L$ is the set of directed edges (links) in the network. The nodes communicate with each other via wireless links. Each node can communicate directly with a subset of other nodes. Assume the network is shared by a set $\mathcal{S}$ of source indexed by $s$. A sequence of connected links $l \in L(s)$ forms a route originating from source $s$, and $f_{l}$ is the link capacity, in terms of the attainable data rate rather than the information-theoretic multiterminal channel capacity on logical link $l$. Since wireless links use a shared medium, they may interfere with each other if they transmit data at the same time. If the interference level between two links is too high, transmissions from those two links will collide, resulting in a failure of the transmission. In the following, we present the detailed interference relation based on the link layer model. In general, the link capacity $f_{l}$ can be represented as the function of power consumption and link access probability in a random access network.

\section{A. Random Access, Power Control and Link Capacity}

In this section, we present the random access protocol with neighborhood link interference information. Define $L_{\text {out }}(v)$ as a set of outgoing links from node $v, L_{i n}(v)$ as a set of incoming links to node $v, t_{l}$ as the transmitter node of link $l$, and $r_{l}$ as the receiver node of link $l$. Assume that the transmission of a link is interfered from the transmission of another link, if the distance between the receiver node of the former and the transmitter node of the latter is less than some threshold [10]. We define $N_{t o}^{I}(l)$ as the set of nodes whose transmission cause interference to the receiver of link $l$, excluding the transmitter node of link $l$ and $L_{\text {from }}^{I}(v)$ as the set of links whose transmission get interfer from the transmission of node $v$, excluding outgoing links form node $v$. Each node $v$ transmits data with a probability $\Im_{v}$. When it determines to transmit data, it chooses one of its outgoing links with probability $\frac{q_{l}}{\Im_{v}}, \forall l \in L_{\text {out }}(v)$ such that $\sum_{l \in L_{\text {out }}(v)} \frac{q_{l}}{\Im_{v}}=1$ and transmits data only on the chosen link. Hence there is no collision among links that have the same transmitter node. Link $l, l \in L_{\text {out }}(v)$, transmits data with the persistence probability $q_{l}$ such that

$$
\sum_{l \in L_{\text {out }}(v)} q_{l}=\Im_{v}, \forall v .
$$

We designate the transmitted power and SINR for the $l$ th link by $p_{l}\left(0 \leq p_{l} \leq p_{l}^{\max }\right)$ and $\gamma_{l}$, respectively. The users' transmit power vector is denoted by $\boldsymbol{p}=\left(p_{1}, \ldots, p_{N}\right)$. We denote the background (receiver) noise within the link's bandwidth by $\sigma_{l}$. In the deterministic formulation of the power control problem for wireless multi-hop networks, the noise power $\sigma_{l}$ is dealt with a constant. We use a "snapshot" model with the assumption that link gains evolute slowly with respect to the SINR evolution. In this problem formulation, the average SINR of the $l$ th link is

$$
\gamma_{l}(\boldsymbol{p}, \boldsymbol{q})=\frac{h_{t_{l} r_{l}} p_{l}}{\sigma_{l}^{2}+\frac{1}{B} \sum_{v \in N_{\text {to }}^{I}(l)} \sum_{k \in L_{\text {out }}(v)} h_{t_{k} r_{l}} p_{k} q_{k}},
$$

where $h_{t_{l} r_{l}}$ is the link gain from the $l$ th link's transmitter $t_{l}$ to its intended receiver $r_{l}$, and $h_{t_{k} r_{l}}$ is the link gain from the $k$ th link's transmitter $t_{k}$ to the $l$ th user's receiver $r_{l}$. We denote the interference plus noise of link $l$ by

$$
\mathcal{I}_{l}\left(\boldsymbol{p}_{-l}, \boldsymbol{q}_{-l}\right)=\sigma_{l}^{2}+\frac{1}{B} \sum_{v \in N_{\text {to }}^{I}(l)} \sum_{k \in L_{\text {out }}(v)} h_{t_{k} r_{l}} p_{k} q_{k} .
$$

A packet is assumed to be received without error if the SINR is above a target SINR $\gamma_{l}^{t g t}$ for the duration of the slot in which it is transmitted. The link capacity is determined by the power level and link access probability based on the following interference-based communication model

$$
f_{l}(\boldsymbol{p}, \boldsymbol{q})= \begin{cases}q_{l} \theta_{l} \log \left(1+\gamma_{l}\right), & \text { if } \gamma_{l} \geq \gamma_{l}^{t g t} \\ 0, & \text { otherwise. }\end{cases}
$$

where $\theta_{l}=\left(1-\sum_{k \in L_{\text {in }}\left(t_{l}\right)} q_{k}\right)\left(1-\sum_{k \in L_{\text {out }}\left(r_{l}\right)} q_{k}\right)$ denotes that both transmitter $t_{l}$ of link $l$ and its intended receiver $r_{l}$ cannot transmit or receive simultaneously.

\section{B. Problem Formulation}

Consider a set of $\mathcal{S}=\{1, \ldots, S\}$ users, where each user injects data into the network and travels through one fixed route. Let $x_{s}$ be the rate that user $s$ injects into the network, and $U_{s}\left(x_{s}\right)$ be the utility function of user $s, L(s)$ be the link set of user $s, \boldsymbol{R}=\left[R_{l, s}\right]$ be the routing matrix, i.e.

$$
R_{l, s}= \begin{cases}1, & \text { if } l \in L(s), \\ 0, & \text { otherwise. }\end{cases}
$$

We now make the following assumptions on the utility function $U_{s}$ for all users $s$.

A1 $U_{s}\left(x_{s}\right)$ is continuous in $x_{s}$ and twice continuously differentiable, non-decreasing and uniformly strictly concave in $x_{s}$, that is, $d U_{s}\left(x_{s}\right) / d x_{s} \geq 0$, $d^{2} U_{s}\left(x_{s}\right) / d x_{s}^{2} \leq-\varepsilon, \varepsilon>0, \forall s$. Moreover, it should satisfy the following condition

$$
G_{s}\left(x_{s}\right)=\frac{d^{2} U_{s}\left(x_{s}\right)}{d x_{s}^{2}} x_{s}+\frac{d U_{s}\left(x_{s}\right)}{d x_{s}} \leq 0 .
$$

A2 Each user $s$ always has data to transmit and has the maximum data rate $x_{s}^{\max }$, i.e., $0<x_{s} \leq x_{s}^{\max }$.

Remark 1: The constraint in (6) demonstrates that the utility must be elastic enough. Note that the condition is not restrictive and holds for the following class of utility functions.

$$
U^{\alpha}(x)= \begin{cases}(1-\alpha)^{-1} x^{1-\alpha} & \text { if } \alpha>1 \\ \log x & \text { if } \alpha=1\end{cases}
$$

This class of utility functions are known to characterize a large class of fairness concepts [10].

Before proceeding, we introduce the following notation:.

$$
\begin{aligned}
\mathcal{X} & =\left\{x_{s}, s \in \mathcal{S} \mid 0<x_{s} \leq x_{s}^{\max }\right\}, \\
\mathcal{Q} & =\left\{q_{l}, l \in \mathcal{L} \mid 0 \leq q_{l} \leq 1\right\}, \\
\mathcal{P} & =\left\{p_{l}, l \in \mathcal{L} \mid 0 \leq p_{l} \leq p_{l}^{\max }\right\}
\end{aligned}
$$

The network utility maximization (NUM) problem is formulated as follows

$$
\begin{array}{cl}
\max _{\substack{\boldsymbol{x} \in \mathcal{X}, \boldsymbol{q} \in \mathcal{Q}, \boldsymbol{p} \in \mathcal{P} \\
\text { subject to }}} & \sum_{s} U_{s}\left(x_{s}\right) \\
& \sum_{s: l \in L(s)} x_{s} \leq q_{l} \theta_{l} \log \left(1+\gamma_{l}\right), \forall l \\
& \gamma_{l} \geq \gamma_{l}^{t g t}, \forall l \\
& \sum_{l \in L(v)} q_{l} \leq 1, \quad \forall v
\end{array}
$$


The first and second sets of constraints ensure that the total source rates of traffic in a link cannot exceed the attainable rate of the link with the required interference constraints. The third set of constraint comes from the fact that the link persistent probabilities and node random access probabilities are non-negative, and that cannot be greater than unity. The objective of this problem is to obtain the optimal persistence probabilities $\boldsymbol{q}$, transmit powers $\boldsymbol{p}$ on links that maximize the network utility. The rate control question therefore represents a joint optimization problem which couples the link persistent probabilities at link layer, transmit powers at the physical layer with the end-to-end source rates at the transport layer. Note that the NUM problem P1 is non-convex and non-separable. The major challenges are the global dependence between the source rates $\boldsymbol{x}$ and link capacities $\boldsymbol{f}$ and the nonlinear couple among link capacities $\boldsymbol{f}$, link persistent probabilities $\boldsymbol{q}$ and link transmit powers $\boldsymbol{p}$. Our primary goal in this paper is to distributively find the jointly and globally optimal solution $\left(\boldsymbol{x}^{*}, \boldsymbol{q}^{*}, \boldsymbol{p}^{*}\right)$ to problem $\mathbf{P 1}$ by breaking down the global dependence and nonlinear couple limitation. The interesting readers can refer to [7], [13], [19] for the recent development in cross-layer decomposition methods for NUM problem.

\section{Distributed Algorithms Design VIA LAYERED \\ DECOMPOSITION METHOD}

In this Section, we first present an equivalent convex formulation by variable substitute method. That is, the non-convex and non-separable programming P1 will be converted into a standard geometric programming problem. Then a general dual decompostion method can be used to seperate the joint design.

\section{A. Equivalent Convex Formulation}

First, we give the following notations. Let $y_{l}=\gamma_{l}$ and log change of variables as shown on the top of the following page. Correspondingly, the domains of these variables by excluding the boundary region are denoted as

$$
\begin{aligned}
& \hat{\mathcal{X}}=\left\{\hat{x}_{s}, s \in \mathcal{S} \mid-M \leq \hat{x}_{s} \leq \log x_{s}^{\max }\right\}, \\
& \hat{\mathcal{Q}}=\left\{\hat{q}_{l}, l \in \mathcal{L} \mid-M \leq \hat{q}_{l} \leq 0\right\} \\
& \hat{\mathcal{P}}=\left\{\hat{p}_{l}, l \in \mathcal{L} \mid-M \leq \hat{p}_{l} \leq \log p_{l}^{\max }\right\}
\end{aligned}
$$

where $M$ is a large positive constant. Let

$$
\begin{aligned}
\hat{U}_{s}\left(\hat{x}_{s}\right) & =U_{s}\left(e^{\hat{x}_{s}}\right) \\
\hat{\mathcal{I}}_{l}\left(\hat{\boldsymbol{p}}_{-l}, \hat{\boldsymbol{q}}_{-l}\right) & =\sigma^{2}+\frac{1}{B} \sum_{v \in N_{\text {to }}^{I}(l)} \sum_{k \in L_{\text {out }}(v)} e^{\left(\hat{h}_{t_{k} r_{l}}+\hat{p}_{k}+\hat{q}_{k}\right)}
\end{aligned}
$$

and the constraint sets in (7) can be rewritten as following with respect to the log change of variables:

$$
\begin{aligned}
\hat{\mathcal{C}}_{l}\left(\hat{\boldsymbol{x}}, \hat{y}_{l}\right) & \leq 0, \quad \hat{\mathcal{D}}_{l}\left(\hat{\boldsymbol{p}}, \hat{\boldsymbol{q}}_{-l}, \hat{y}_{l}\right) \leq 0, \\
\hat{\mathcal{E}}_{l}\left(\hat{\boldsymbol{p}}, \hat{\boldsymbol{q}}_{-l}\right) & \leq 0, \quad \hat{\mathcal{F}}_{v}(\hat{\boldsymbol{q}}) \leq 0
\end{aligned}
$$

where

$$
\begin{aligned}
& \hat{\mathcal{C}}_{l}\left(\hat{\boldsymbol{x}}, \hat{y}_{l}\right)=\log \left(\sum_{s \in \mathcal{S}(l)} e^{\hat{x}_{s}}\right)-\log \left(\theta_{l} \log \left(1+e^{\hat{y}_{l}}\right) \hat{q}_{l}\right), \\
& \hat{\mathcal{D}}_{l}\left(\hat{\boldsymbol{p}}, \hat{\boldsymbol{q}}_{-l}, \hat{y}_{l}\right)=\hat{y}_{l}+\log \hat{\mathcal{I}}_{l}\left(\hat{\boldsymbol{p}}_{-l}, \hat{\boldsymbol{q}}_{-l}\right)-\hat{p}_{l}-\hat{h}_{t_{l} r_{l},} \\
& \hat{\mathcal{E}}_{l}\left(\hat{\boldsymbol{p}}, \hat{\boldsymbol{q}}_{-l}\right)=\log \gamma_{l}^{t g t}+\log \hat{\mathcal{I}}_{l}\left(\hat{\boldsymbol{p}}_{-l}, \hat{\boldsymbol{q}}_{-l}\right)-\hat{p}_{l}-\hat{h}_{t_{l} r_{l}},
\end{aligned}
$$

$$
\hat{\mathcal{F}}_{v}(\hat{\boldsymbol{q}})=\log \left(\sum_{l \in L(v)} e^{\hat{q}_{l}}\right) .
$$

Therefore, we have the following Theorem.

Theorem 1: The non-convex programming problem $\mathbf{P 1}$ can be equivalently converted to the following nonlinear programming problem $\mathbf{P 2}$

$$
\begin{array}{ll}
\max _{\hat{\boldsymbol{x}} \in \hat{\mathcal{X}}, \hat{\boldsymbol{q}} \in \hat{\mathcal{Q}}, \boldsymbol{p} \in \hat{\mathcal{P}}} & \sum_{s} \hat{U}_{s}\left(\hat{x}_{s}\right) \\
\text { subject to } & (8)
\end{array}
$$

Moreover, assume $q_{l} \leq \sum_{k \in L_{\text {out }}(v)} q_{k}, \forall l \in L_{\text {in }}(v), \forall v$, and $q_{l} \leq \sum_{k \in L_{\text {in }}(v)} q_{k}, \forall l \in L_{\text {out }}(v), \forall v$, the problem $\mathbf{P 2}$ is convex programming.

Proof: See the Appdenix I.

Remark 2: The assumptions $q_{l} \leq \sum_{k \in L_{\text {out }}(v)} q_{k}, \forall l \in$ $L_{\text {in }}(v), \forall v$, and $q_{l} \leq \sum_{k \in L_{\text {in }}(v)} q_{k}, \forall l \in L_{\text {out }}(v), \forall v$ pose additional constraints for the link access, i.e. for all the node $v$, any incoming link's access probability of node $v$ is no larger than the sum of its outgoing links' access probability and any outgoing link's access probability of node $v$ is no larger than the sum of its incoming links' access probability.

\section{B. Dual Decomposition}

Consider the dual to the primal problem (9)

$$
\min _{\boldsymbol{\lambda} \succeq \mathbf{0}, \boldsymbol{\mu} \succeq \mathbf{0}} D(\boldsymbol{\lambda}, \boldsymbol{\mu})
$$

with partial dual function

$$
\begin{aligned}
D(\boldsymbol{\lambda}, \boldsymbol{\mu})=\max _{\hat{\boldsymbol{x}} \in \hat{\mathcal{X}}, \hat{\boldsymbol{q}} \in \hat{\mathcal{Q}}, \boldsymbol{p} \in \hat{\mathcal{P}}} & \tilde{\Pi} \\
\text { subject to } & \hat{\mathcal{E}}_{l}\left(\hat{\boldsymbol{p}}, \hat{\boldsymbol{q}}_{-l}\right) \leq 0, \forall l \\
& \hat{\mathcal{F}}_{v}(\hat{\boldsymbol{q}}) \leq 0, \forall v
\end{aligned}
$$

where $\tilde{\Pi}=\sum_{s} \hat{U}_{s}\left(\hat{x}_{s}\right)-\sum_{l} \lambda_{l} \hat{\mathcal{C}}_{l}\left(\hat{\boldsymbol{x}}, \hat{y}_{l}\right)-$ $\sum_{l} \mu_{l} \hat{\mathcal{D}}_{l}\left(\hat{\boldsymbol{p}}, \hat{\boldsymbol{q}}_{-l}, \hat{y}_{l}\right)$. Note that we relax only the first and second constraints in (9) by introducing Lagrangian multiplier $\lambda_{l}$ and $\mu_{l}$ for link $l \in \mathcal{L}$. If $\hat{\Pi}^{*}$ and $D^{*}$ denote the optimal value of (9) and (10) respectively, we have $D^{*} \geq \hat{\Pi}^{*}$ with the weak duality theorem. Since $\mathbf{P 2}$ is a convex programming, there is no duality gap, i.e. $D^{*}=\hat{\Pi}^{*}$.

The maximization in (11) can be decomposed into the following three subproblems

$$
D_{1}(\boldsymbol{\lambda})=\max _{\hat{\boldsymbol{x}} \in \hat{\mathcal{X}}}\left\{\sum_{s} \hat{U}_{s}\left(\hat{x}_{s}\right)-\sum_{l} \lambda_{l} \log \left(\sum_{s: l \in L(s)} e^{\hat{x}_{s}}\right)\right\},
$$

$$
D_{2}(\boldsymbol{\lambda}, \boldsymbol{\mu})=\max _{\hat{\boldsymbol{y}}}\left\{\sum_{l}\left(\lambda_{l} \log \left(\log \left(1+e^{\hat{y}_{l}}\right)\right)-\mu_{l} \hat{y}_{l}\right)\right\},
$$

and

$$
\begin{aligned}
D_{3}(\boldsymbol{\lambda}, \boldsymbol{\mu})=\max _{\hat{\boldsymbol{q}} \in \hat{\mathcal{Q}}, \hat{\boldsymbol{p}} \in \hat{\mathcal{P}}} & \Xi(\hat{\boldsymbol{p}}, \hat{\boldsymbol{q}}) \\
\text { subject to } & \hat{\mathcal{E}}_{l}\left(\hat{\boldsymbol{p}}, \hat{\boldsymbol{q}}_{-l}\right) \leq 0, \forall l \\
& \hat{\mathcal{F}}_{v}(\hat{\boldsymbol{q}}) \leq 0, \forall v
\end{aligned}
$$

where

$\Xi(\hat{\boldsymbol{p}}, \hat{\boldsymbol{q}})=\sum_{l}\left\{\lambda_{l} \hat{q}_{l}+\lambda_{l} \log \hat{\theta}_{l}-\mu_{l}\left(\hat{\mathcal{D}}_{l}\left(\hat{\boldsymbol{p}}, \hat{\boldsymbol{q}}_{-l}, \hat{y}_{l}\right)-\hat{y}_{l}\right)\right\}$. 


$$
\hat{x}_{s}=\log x_{s}, \hat{q}_{l}=\log q_{l}, \hat{y}_{l}=\log y_{l}, \hat{p}_{l}=\log p_{l}, \hat{h}_{t_{k} r_{l}}=\log h_{t_{k} r_{l}}
$$

Remark 3: With the dual decomposition method, the system optimization problem can be decomposed by rate control problem in (12) and resource allocation problem in (14), which is to determine the link's access probability and power level. The second subproblem (13) can be viewed as an interface between rate control subproblem and resource allocation subproblem, which is introduced by the auxiliary variable $\hat{\boldsymbol{y}}$. Thus the cross-layer optimization problem P2 decomposes into separate optimization problems of transport layer and link/physical layers, respectively, and they interact through congestion prices $\lambda_{l}$ and link capacity estimate prices $\mu_{l}$.

Due to the function $G(\hat{\boldsymbol{x}}, \boldsymbol{\lambda})=\sum_{s} \hat{U}_{s}\left(\hat{x}_{s}\right)-$ $\sum_{l} \lambda_{l} \log \left(\sum_{s \in \mathcal{S}(l)} e^{\hat{x}_{s}}\right)$ is concave with respect to $\hat{\boldsymbol{x}}$, we can solve the subproblem in (12) by the first-order optimal condition $\frac{\partial G(\hat{\boldsymbol{x}, \boldsymbol{\lambda}})}{\partial \hat{x}_{s}}=\hat{U}_{s}^{\prime}\left(\hat{x}_{s}\right)-\lambda_{s} x_{s}=0$, where $\lambda_{s}=$ $\sum_{l \in L(s)} \lambda_{l} / \sum_{s \in \mathcal{S}(l)} x_{s}$. Consider the proportional fairness utility $U\left(x_{s}\right)=\log x_{s}$, the end-to-end rate is adjusted by

$$
x_{s}^{(t+1)}=\left[1 / \lambda_{s}^{(t)}\right]_{0}^{x_{s}^{\max }},
$$

where $[a]_{c}^{b}=\max \{\min \{a, b\}, c\}$.

The second subproblem (13) can be viewed as an interface between network subproblem and resource subproblem, which is introduced by the auxiliary variable $\hat{\boldsymbol{y}}$. Solving the second subproblem (13), we have the following relations

$$
\mu_{l}=\lambda_{l} \frac{e^{\hat{y}_{l}}}{\log \left(1+e^{\hat{y}_{l}}\right)\left(1+e^{\hat{\hat{y}_{l}}}\right)}, \forall l \in \mathcal{L} .
$$

The third subproblem (14) is the resource allocation problem to determine the link's access probability and power level. It is easy to verify the following proposition based on the subgradient definition in [22].

Proposition 1: Let $\boldsymbol{\lambda} \succeq \mathbf{0}, \boldsymbol{\mu} \succeq \mathbf{0}$ be given, and $\hat{\boldsymbol{x}}, \hat{\boldsymbol{q}}, \hat{\boldsymbol{p}}$ be the associated optimal solutions to the network optimal problem (12) and the resource allocation problem (14), respectively. Then $\hat{\mathcal{C}}_{l}\left(\hat{\boldsymbol{x}}, \hat{y}_{l}\right)$ and $\hat{\mathcal{D}}_{l}\left(\hat{\boldsymbol{p}}, \hat{\boldsymbol{q}}_{-l}, \hat{y}_{l}\right)$ are the subgradient of dual function $D(\boldsymbol{\lambda}, \boldsymbol{\mu})$ at point $\boldsymbol{\lambda}$ and $\boldsymbol{\mu}$, respectively.

The dual problem (10) can then be solved using subgradient projection method, where the Lagrangian multipliers $\lambda_{l}$, and $\mu_{l}$ are adjusted in the direction opposite to the subgradients $\nabla_{\boldsymbol{\lambda}} D(\boldsymbol{\lambda}, \boldsymbol{\mu})$, and $\nabla_{\boldsymbol{\mu}} D(\boldsymbol{\lambda}, \boldsymbol{\mu})$.

$$
\begin{aligned}
\lambda_{l}^{(t+1)} & =\left[\lambda_{l}^{(t)}-\beta^{(t)} \frac{\partial D}{\partial \lambda_{l}}\left(\boldsymbol{\lambda}^{(t)}, \boldsymbol{\mu}^{(t)}\right)\right]^{+} \\
& =\left[\lambda_{l}^{(t)}+\beta^{(t)} \hat{\mathcal{C}}_{l}\left(\hat{\boldsymbol{x}}^{(t)}, \hat{y}_{l}^{(t)}\right)\right]^{+} \\
\mu_{l}^{(t+1)} & =\left[\mu_{l}^{(t)}-\beta^{(t)} \frac{\partial D}{\partial \mu_{l}}\left(\boldsymbol{\lambda}^{(t)}, \boldsymbol{\mu}^{(t)}\right)\right]^{+} \\
& =\left[\mu_{l}^{(t)}+\beta^{(t)} \hat{\mathcal{D}}_{l}\left(\hat{\boldsymbol{p}}^{(t)}, \hat{\boldsymbol{q}}_{-l}^{(t)}, \hat{y}_{l}^{(t)}\right)\right]^{+} .
\end{aligned}
$$

Herein, $t$ is the iteration number, $\beta^{(t)}$ is the positive scalar step size, and ' + ' denotes the projection onto the set $\Re^{+}$of non-negative real numbers.
From Eq. (16) and (18), we have the following closedrelations

$$
\left\{\begin{array}{l}
\hat{y}_{l}^{(t+1)}=\log \gamma_{l}^{(t)} \\
\mu_{l}^{(t+1)}=\lambda_{l}^{(t)} \frac{\gamma_{l}^{(t)}}{\log \left(1+\gamma_{l}^{(t)}\right)\left(1+\gamma_{l}^{(t)}\right)}
\end{array}, \forall l \in \mathcal{L} .\right.
$$

The above dual decomposition method achieves a distributed solution for the joint rate control, random access and power control design, where at the transport layer sources $s$ individually adjust their rates according to the congestion price, and link prices according to formula (17). At the link/physical layer, the link $l$ solve the resource allocation problem (14) to determine their random access probabilities and power levels.

\section{Joint Random Access and Power Control Design}

It is worth noting that the boundary conditions $\hat{\mathcal{E}}_{l}\left(\hat{\boldsymbol{p}}, \hat{\boldsymbol{q}}_{-l}\right)=0$ and $\hat{\mathcal{F}}_{v}(\hat{\boldsymbol{q}})=0$ are not the optimal points for the subproblem (14). Therefore, the general dual decomposition method with gradient (subgradient) algorithm is not suitable for solving subproblem (14). In the following, we introduce the penalty method to solve the joint random access and power control design problem. A penalty function should impose a positive penalty at infeasible points and no penalty at feasible points. We apply the non-smooth penalty function method and consider the following problem:

$$
\underset{\hat{\boldsymbol{q}} \in \hat{\mathcal{Q}}, \hat{\boldsymbol{p}} \in \hat{\mathcal{P}}}{\operatorname{maximize}} \Psi(\hat{\boldsymbol{p}}, \hat{\boldsymbol{q}})
$$

where

$$
\begin{aligned}
\Psi(\hat{\boldsymbol{p}}, \hat{\boldsymbol{q}})= & \Xi(\hat{\boldsymbol{p}}, \hat{\boldsymbol{q}})-\kappa \sum_{l} \max \left\{\hat{\mathcal{E}}_{l}\left(\hat{\boldsymbol{p}}, \hat{\boldsymbol{q}}_{-l}\right), 0\right\} \\
& -\kappa \sum_{v} \max \left\{\hat{\mathcal{F}}_{v}(\hat{\boldsymbol{q}}), 0\right\},
\end{aligned}
$$

and $\kappa$ is a positive penalty constant. Since the objective function of problem (14) is concave, problem (20) is convex optimization with simple, decoupled constraints, which can be solved by using a subgradient projection algorithm. We can easily show that

$$
\begin{aligned}
\frac{\partial \Psi(\hat{\boldsymbol{p}}, \hat{\boldsymbol{q}})}{\partial \hat{q}_{l}}= & \lambda_{l}-\sum_{j: l \in L_{\text {in }}\left(t_{j}\right)} \lambda_{j} \frac{q_{l}}{1-\sum_{k \in L_{\text {in }}\left(t_{j}\right)} q_{k}} \\
& -\sum_{k \in L_{\text {from }}^{I}\left(t_{l}\right)} h_{t_{l} r_{k}} m_{k} p_{l} q_{l}-\frac{\kappa \delta_{t_{l}} q_{l}}{\sum_{k \in L\left(t_{l}\right)} q_{k}} \\
& -\sum_{j: l \in L_{\text {out }}\left(r_{j}\right)} \lambda_{j} \frac{q_{l}}{1-\sum_{k \in L_{\text {out }}\left(r_{j}\right)} q_{k}}, \quad(21) \\
\frac{\partial \Psi(\hat{\boldsymbol{p}}, \hat{\boldsymbol{q}})}{\partial \hat{p}_{l}}= & \left(\mu_{l}+\kappa \varepsilon_{l}\right)-\sum_{k \in L_{\text {from }}^{I}\left(t_{l}\right)} h_{t_{l} r_{k} m_{k} p_{l} q_{l}}
\end{aligned}
$$

where

$$
m_{k}=\frac{\left(\mu_{k}+\kappa \varepsilon_{k}\right) \gamma_{k}}{B h_{t_{k} r_{k}} p_{k}}
$$




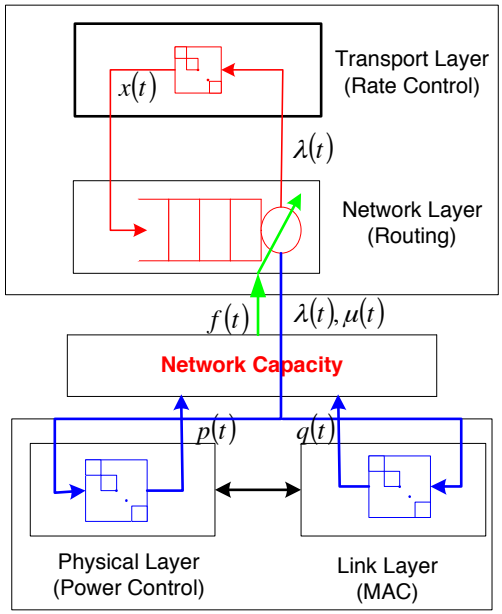

Fig. 5. The closed-loop control structure for cross-layer design.

$$
\varepsilon_{l}=\left\{\begin{array}{ll}
0, & \text { if } \gamma_{l} \geq \gamma_{l}^{t g t} \\
1, & \text { otherwise }
\end{array}, l \in \mathcal{L},\right.
$$

and

$$
\delta_{v}=\left\{\begin{array}{ll}
0, & \text { if } \sum_{k \in L_{\text {out }}(v)} q_{k} \leq 1 \\
1, & \text { otherwise }
\end{array}, v \in \mathcal{N} .\right.
$$

Then, the subgradient projection algorithm that solves problem (20) is obtained as follows. On each logical link $l$, transmission is decided to take place with probability

$$
q_{l}^{(t+1)}=\left[e^{\left(\log q_{l}^{(t)}+\left.\alpha^{(t)} \frac{\partial \Psi(\hat{p}, \hat{q})}{\partial q_{l}}\right|_{\hat{p}=\hat{p}(t), \hat{q}=\hat{q}(t)}\right)}\right]_{0}^{1},
$$

and the transmission power control level is adjusted

$$
p_{l}(t+1)=\left[e^{\left(\log p_{l}(t)+\left.\alpha^{(t)} \frac{\partial \Psi(\hat{\boldsymbol{p}}, \hat{\boldsymbol{q}})}{\partial \hat{p}_{l}}\right|_{\hat{\boldsymbol{p}}=\hat{\boldsymbol{p}}(t), \hat{\boldsymbol{q}}=\hat{\boldsymbol{q}}(t)}\right)}\right]_{0}^{p_{l}^{\max }},
$$

where $\alpha^{(t)}$ is step size.

Remark 4: We present some intuitive arguments on joint random access and power control design in (26)-(27). Eq. (21)-(22) show each link needs the broadcast message $m_{k}$ and congestion prices $\lambda_{j}$ of its receiver's outgoing links at each iterative. Note that the message $m_{k}$ can be computed with the local measurement information $\gamma_{k}$ and $h_{t_{k} r_{k}} p_{k}$. Intuitively, if the local link congestion status is high, the random access probability should increase. If the congestion conditions on other links are high, the random access probability and power level should decrease in order to reduce interference on those links. The update in (26)-(27) is consistent with the intuition. Note that $\lambda_{l}>\mu_{l}$ due to $\gamma_{l}^{(t)} / \log \left(1+\gamma_{l}^{(t)}\right)\left(1+\gamma_{l}^{(t)}\right)<$ 1 , thus the random access probability may increase more aggressively than the power level when the link congestion is increasing. This characteristic leads to energy efficiency in our joint design. Another characteristic of this design is that the power update needs the link access probability information. The closed-loop structure of our proposed cross-layer design is shown in Fig. 5.

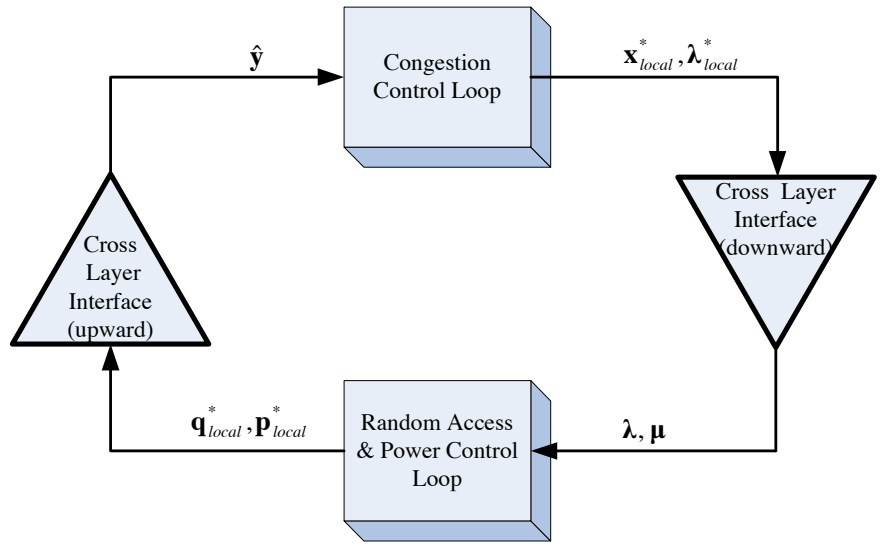

Fig. 6. Algorithm implementation with strict time scheduling.

\section{AlgORITHM IMPLEMENTATION ISSUES}

In this section, we describe in detail a distributed implementation of the dual based algorithm to solve the NUM problem (7). Using the properties of standard gradient or subgradient method, we can show the convergence of the rate control algorithm with fixed $\hat{\boldsymbol{y}}$ and joint random access and power control algorithm with fixed $\boldsymbol{\lambda}, \boldsymbol{\mu}$. To guarantee the stability of the entire protocol stack, however, this implementation will pose a strict time scheduling. That is to say, the rate control loop is scheduled to run when the joint random access and power control loop converges to a local optimum point as well as the joint random access and power control loop. The algorithm implementation with strict time scheduling is shown in Fig. 6.

To reduce the implementation complexity, we present an asynchronous implementation for the cross-layer design. Firstly, we show the subalgorithm implementation in transport layer, cross-layer interface and link/physical layer, respectively.

\section{A. Rate Control at Transport Layer}

Let $\mathcal{T}_{r} \subseteq\{1,2, \cdots\}$ be a set of times at which source $s \in \mathcal{S}$ update their transport rates and link $l \in \mathcal{L}$ update their congestion prices. Given the parameter estimate $\hat{\boldsymbol{y}}$ from the cross-layer interface, the rate control algorithm at transport layer is summarized in SA 1.

\section{B. Cross-Layer Interface}

Let $\mathcal{T}_{i} \subseteq\{1,2, \cdots\}$ be a set of times at which link $l \in \mathcal{L}$ update their link prices and auxiliary parameters estimate. In the cross-layer interface, there are two functions. One is to provide the information of estimate prices to the link/physical layer, which is called the downward information procedure. The other is in charge of sending the estimate parameters to transport layer, which is called the upward information procedure. The algorithm at the cross-layer interface is summarized as follows.

\section{Joint Random Access and Power Control}

Let $\mathcal{T}_{j} \subseteq\{1,2, \cdots\}$ be a set of times at which link $l \in \mathcal{L}$ update their random access probabilities and power levels. 


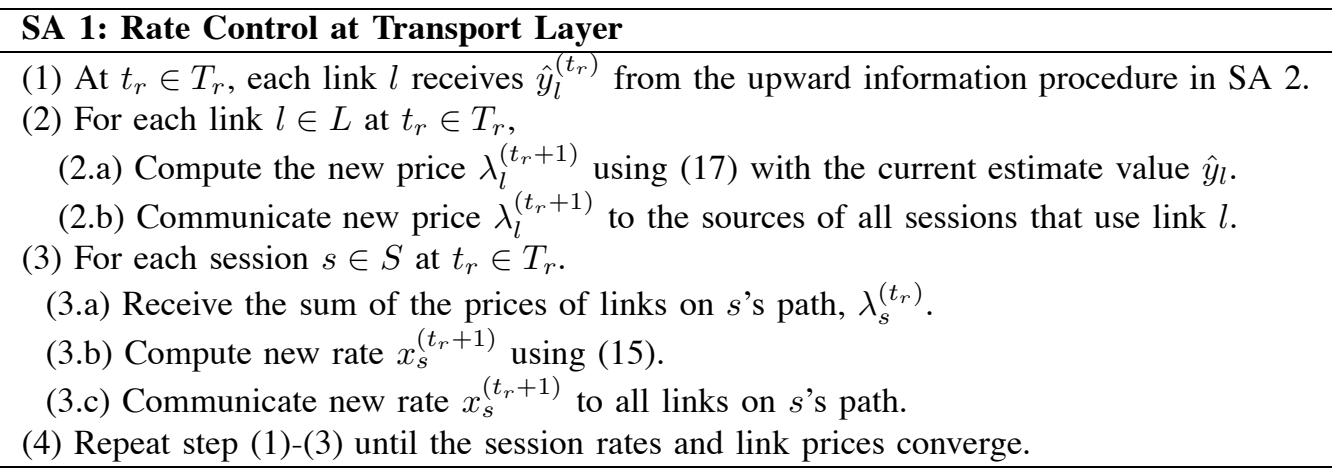

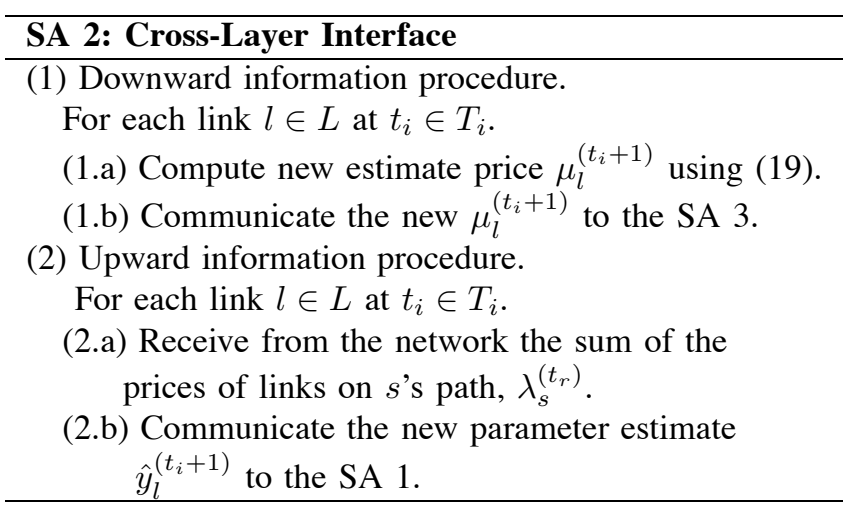

SA 3: Joint Random Access and Power Control

(1) For each link $l \in L$, receive the estimate price $\mu_{l}^{\left(t_{j}\right)}$ from the downward information procedure in SA 2.

(2) At $t_{j} \in T_{j}$, each node $v$ computes $\delta_{v}^{\left(t_{j}\right)}$ with (25).

(3) For each link $l \in L$ at $t_{j} \in T_{j}$,

(3.a) According to the SINR measurment $\gamma_{l}$ at time $t_{j}$, compute $\varepsilon_{l}^{\left(t_{j}\right)}$ with (24).

(3.b) Compute new random access probability $q_{l}^{\left(t_{j}+1\right)}$ using (26).

(3.c) Compute new power level $p_{l}^{\left(t_{j}+1\right)}$ using (27).

(4) Repeat step (1)-(3) until the random access probabilities and power levels converge.

Given the estimate price $\boldsymbol{\mu}$ from the cross-layer interface, the algorithm for joint random access and power control design at the link/physical layer using subgradient algorithms are summarized as follows.

\section{Asynchronous Algorithm Implementation}

The asynchronous algorithm implementation for the crosslayer design is summarized in Algorithm 1.

The diagram of asynchronous algorithm implementation is shown in Fig. 7.

If $\mathcal{T}_{r}=\mathcal{T}_{i}=\mathcal{T}_{j}$, the Algorithm 1 is reduced to the following JOCRP algorithm.

Jointly Optimal Congestion-Control, Random-Access and Power-Control (JOCRP) Algorithm: During each time slot $t$, the following four steps are carried out simultaneously until convergence.

(1) cross-layer Interface: parameter estimate and link/node information computing.

\section{Algorithm 1: Asychronous Algorithm for Joint Design with Dual Decomposition Method}

(1) Initial. For any link $l \in L$, choose the initial random access probability $q_{l}$ and power level $p_{l}$.

(2) For $t_{r} \in T_{r}$, the subalgorithm 1 is scheduled to run for end-to-end rate control.

(3) For $t_{i} \in T_{i}$, the subalgorithm 2 is scheduled to parameter estimate.

(4) For $t_{j} \in T_{j}$, the subalgorithm 3 is scheduled to run for the update of random access probability and power level.
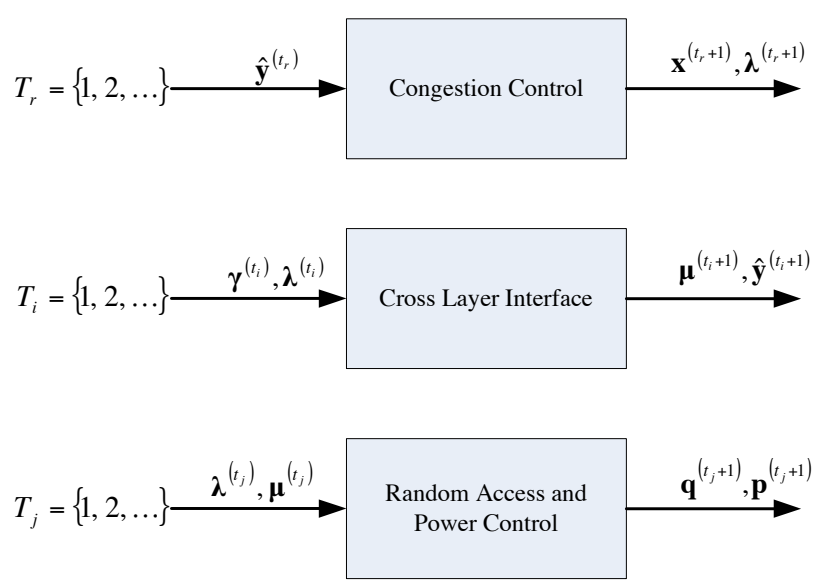

Fig. 7. Asynchronous algorithm implementation diagram.

1.1) According to the measured link SINR $\gamma_{l}^{(t)}$, each link $l \in \mathcal{L}$ updates its auxiliary variable $\hat{y}_{l}^{(t+1)}=\log \gamma_{l}^{(t)}$, and the estimate price $\mu_{l}^{(t+1)}$ using Eq. (19).

1.2) Based on the measured SINR $\gamma_{l}^{(t)}$, each link $l \in \mathcal{L}$ computes the QoS penalty parameter $\varepsilon_{l}^{(t)}$ according to Eq. (24) and each node $v \in \mathcal{N}$ computes the $\delta_{v}^{(t)}$ with Eq. (25).

1.3) Each link $l \in \mathcal{L}$ computes $m_{l}^{(t)}$ with Eq. (23) and communicate $m_{l}^{(t)}$ to all links $k: t_{k} \in N_{t o}^{I}(l)$.

(2) Each link $l \in \mathcal{L}$ updates the congestion price $\lambda_{l}^{(t+1)}$ with Eq. (17) and communicates new price $\lambda_{l}^{(t+1)}$ to the sources of all sessions that use link $l$.

(3) Rate control: each source $s \in \mathcal{S}$ receives the sum of the prices of links on $s$ 's path, $\lambda_{s}$, from the network and computes the new end-to-end rate $x_{s}^{(t+1)}$ according to Eq. (15).

(4) Joint random access and power control design: given 


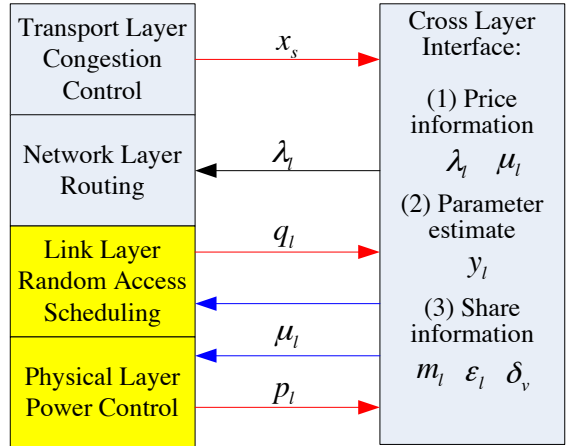

Fig. 8. Protocol stack for the cross-layer design.

the and congestion price $\lambda_{l}^{(t)}$ and estimate price $\mu_{l}^{(t)}$, each link $l \in \mathcal{L}$ updates its random access probability and power level according to the convex optimization problem in (14).

4.1) Link layer scheduling: each link $l \in \mathcal{L}$ updates its random access probability with Eq. (26).

4.2) Power Control: each link $l \in \mathcal{L}$ updates its power level with Eq. (27).

Remark 5: The JOCRP algorithm is a distributed implementation for rate control, random access and power control, which maintains the architectural modularity among layers. It only needs an additional cross-layer interface for parameter estimate and sharing information. In the joint random access and power control (26)-(27), each link needs to communication the message $m_{l}$ to its neighboring nodes. This can be implemented by message passing (broadcast or inquire). Let $\mathfrak{L}$, denote the maximum $\left|L_{\text {from }}^{I}\left(t_{l}\right)\right|, \forall l \in \mathcal{L}$ in the network, the communication overhead for joint random access and power control design is $O(\mathfrak{L}|\mathcal{L}|)$ in each period $t$. Thus, our design has low communication overhead.

Remark 6: Our proposed rate control is an end-to-end scheme, which is the same with the general TCP/AQM framework. As shown in Eq. (17) and (15), we use a rate-based merit to compute congestion price. Compared with the conventional queue-based method, the computing overhead may increase a little due to the log operation.

In the following, we prove the convergence property of our proposed JOCRP algorithm using the results on the convergence of the subgradient method [1], [4]. The proof of the main result is deferred to Appendix II.

Theorem 2: Given small enough positive constants $\alpha, \beta$ and large enough positive constant $\kappa$, the distributed JOCRP algorithm converges statistically to the global optimum of the JCRP problem (7).

\section{Simulation Investigation}

In this section, we investigate the performance of the proposed distributed algorithm. The network that we consider is composed of nine nodes and six links shown in Fig. 9. Assuming that transmissions from two links interfere with each other, if the distance between the transmitter node of one link and the receiver node of the other link is less than $2 d$.

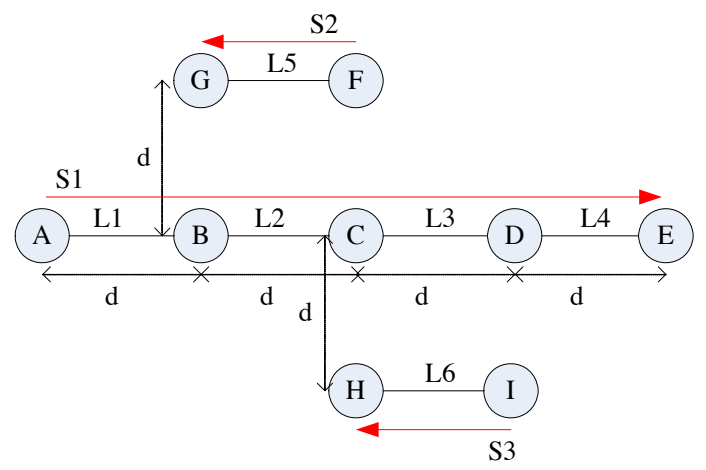

Fig. 9. Physical and logical topologies for the simulation.

We consider the case of end-to-end throughput utility ( $\omega=$ 1) and proportional fairness, i.e. $U_{s}\left(x_{s}\right)=\log x_{s}$. Herein, $B=128, \sigma^{2}=5 \times 10^{-13}, h_{t_{k} r_{l}}=0.097 / d_{t_{k} r_{l}}^{3}$. In this simulation, we compare our proposed JOCRP algorithm with the dual-based Algorithm in [23]. We set $\alpha=0.0001, \beta=$ $0.1, \kappa=1000$, and $\gamma^{t g t}=50$ for JOCRP algorithm. The solutions obtained through JOCRP algorithm and the dualbased Algorithm in [23] are presented in Table I. The globally optimal solutions solved by MATLAB are presented in Table I too. Our aim is to design a network protocol to support end-to-end flow as much as possible with reasonable energy consumption. Therefore, from the total throughput utility comparison, the solutions verify that the JOCRP algorithm achieves the globally optimal solutions. Moreover, our proposed JOCRP algorithm have a desirable energy efficiency property. Fig. 10 shows how the link power levels, link random access probabilities, end-to-end rate, and the aggregate utility converge when the JOCRP algorithm with interference-based communication model is used. It can be seen from the plots that after about 5000 iterations, all the variables are quite close to their globally optimal values. The converge speed is about 3000 iterations for dual-based Algorithm in [23]. Note that $J O C R P$ algorithm is joint three layers design, the convergence times thus is reasonable.

\section{CONCLUSION}

We formulate the end-to-end rate control problem in a random access networks as an optimization problem underlying interference-based communication model. A method combined with geometric programming and variable substitute is proposed to convert the non-convex, non-separable nonlinear programming into convex programming problem. Using dual decomposition techniques, we present a distributed joint random access control and power control algorithm that couples with the existing TCP congestion control algorithms to increase end-to-end throughput and energy efficiency of multi-hop transmissions in wireless multi-hop networks. The proposed JOCRP algorithm can converge to the global optimum. Even we need a merge of random access control and power control and cross-layer interface to share the information, the architectural modularity among layers is maintained as well. The simulation results also validate our analytical observations. 
TABLE I

The Solutions of JOCRP Algorithm , DuAl-BASEd Algorithm In [23] AND Optimal Solutions

\begin{tabular}{c|c|c|c|c|c|c}
\hline \hline Variables & $q_{1}$ & $q_{2}$ & $q_{3}$ & $q_{4}$ & $q_{5}$ & $q_{6}$ \\
\hline JOCRP Algorithm & $\mathbf{0 . 2 6 7 5}$ & $\mathbf{0 . 3 8 9 4}$ & $\mathbf{0 . 3 6 0 0}$ & $\mathbf{0 . 2 1 9 3}$ & $\mathbf{1 . 0 0 0 0}$ & $\mathbf{1 . 0 0 0 0}$ \\
\hline Dual-based Algorithm in [23] & 0.0669 & 0.1383 & 0.1303 & 0.0573 & 0.5288 & 0.5264 \\
\hline Optimal Solutions & 0.2021 & 0.3200 & 0.3202 & 0.2029 & 1.0000 & 1.0000 \\
\hline \hline Variables & $p_{1}$ & $p_{2}$ & $p_{3}$ & $p_{4}$ & $p_{5}$ & $p_{6}$ \\
\hline JOCRP Algorithm & $\mathbf{0 . 0 9 3 5}$ & $\mathbf{0 . 1 0 5 0}$ & $\mathbf{0 . 1 1 2 2}$ & $\mathbf{0 . 1 0 4 3}$ & $\mathbf{0 . 0 6 9 0}$ & $\mathbf{0 . 0 8 0 5}$ \\
\hline Dual-based Algorithm in [23] & 0.3000 & 0.3000 & 0.3000 & 0.3000 & 0.3000 & 0.3000 \\
\hline Optimal Solutions & 0.1719 & 0.1643 & 0.1500 & 0.1078 & 0.3000 & 0.2773 \\
\hline \hline Variables & & $x 1$ & $x 2$ & $x 3$ & total utility & utility/power \\
\hline JOCRP Algorithm & & $\mathbf{0 . 8 5 3 9}$ & $\mathbf{4 . 9 3 5 9}$ & $\mathbf{5 . 0 9 4 6}$ & $\mathbf{3 . 0 6 6 8}$ & $\mathbf{1 0 . 9 8 1 8}$ \\
\hline Dual-based Algorithm in [23] & theory & 0.2591 & 4.0572 & 4.0801 & 1.4561 & 3.3520 \\
& practice & 0.3253 & 3.3913 & 3.3978 & 1.3213 & 3.0417 \\
\hline Optimal Solutions & & 0.6824 & 6.1453 & 6.1671 & 3.2519 & 4.4515 \\
\hline \hline
\end{tabular}

\section{APPENDIX A \\ PROOF OF THEOREM 1}

Proof: Introduce a auxiliary variable $y_{l}$ for the user's SINR $\gamma_{l}$, the first constraint in optimization problem (7) can be expressed as the following two constraints: $\sum_{s \in \mathcal{S}(l)} x_{s} \leq$ $q_{l} \theta_{l} \log \left(1+y_{l}\right), \forall l$ and $y_{l}=\frac{h_{t_{l} r_{l}} p_{l}}{\mathcal{I}_{l}\left(\boldsymbol{p}_{-l}, \boldsymbol{q}_{-l}\right)}, \forall l$. Without loss of generality, we can replace the equality in the second constraint with an inequality and rewrite the constraints with standard geometrical programming form:

$$
\begin{array}{cl}
\max _{\boldsymbol{x} \in \mathcal{X}, \boldsymbol{q} \in \mathcal{Q}, \boldsymbol{p} \in \mathcal{P}} & \sum_{s} U_{s}\left(x_{s}\right) \\
\text { subject to } & \left(q_{l} \theta_{l} \log \left(1+y_{l}\right)\right)^{-1} \sum_{s \in \mathcal{S}(l)} x_{s} \leq 1, \forall l \\
& y_{l} \mathcal{I}_{l}\left(\boldsymbol{p}_{-l}, \boldsymbol{q}_{-l}\right)\left(h_{t_{l} r_{l}} p_{l}\right)^{-1} \leq 1, \forall l \\
& \gamma_{l}^{\text {tgt }} \gamma_{l}^{-1} \leq 1, \forall l \\
& \sum_{l \in L_{\text {out }}(v)} q_{l} \leq 1, \quad \forall v
\end{array}
$$

The next step of problem transformation is to take the log of both sides of the constraints in problem (28) and this reformulation turns the problem into P2. Based on the constraints for the curvatures of $U_{s}$ in Assumption A1, it is easy to verify that the utility function $\hat{U}_{s}\left(\hat{x}_{s}\right)$ is concave with respect to $\hat{x}_{s}$. To show problem $\mathbf{P 2}$ is convex programming, it only needs to proof the convexity of constraints set in (9). We can get the following derivative

$$
\begin{gathered}
\quad \frac{\partial^{2} \log \left(\log \left(1+e^{\hat{y}_{l}}\right)\right)}{\partial \hat{y}_{l}^{2}} \\
=\frac{y_{l}}{\left(1+y_{l}\right)^{2} \log \left(1+y_{l}\right)}\left(1-\frac{y_{l}}{\log \left(1+y_{l}\right)}\right), \\
= \begin{cases}\frac{q^{2} \log \left(1-\sum_{k \in L_{i n}\left(t_{l}\right)} e^{\hat{q}_{k}}\right)}{\partial \hat{q}_{j} \partial \hat{q}_{i}}\left(\frac{q_{j}}{1-q_{i n}\left(t_{l}\right)}\left(\frac{q_{i n}\left(t_{l}\right)}{1-q_{j}}-1\right)\right. & \text { if } j \in L_{i n}\left(t_{l}\right), i=j \\
-\frac{\left.q_{i n}\left(t_{l}\right)\right)^{2}}{\left(1-q_{i n}\right.} & \text { if } i, j \in L_{i n}\left(t_{l}\right), i \neq j \\
0 & \text { otherwise }\end{cases}
\end{gathered}
$$

where $q_{i n}\left(t_{l}\right)=\sum_{k \in L_{i n}\left(t_{l}\right)} q_{k}$. Due to $y_{l} \geq \log \left(1+y_{l}\right)$, $\forall y_{l} \geq 0$, then $\log \left(\log \left(1+e^{\hat{y} l}\right)\right)$ is a concave function for $\hat{y}_{l}$. Based on $\sum_{k \in L_{\text {out }}\left(t_{l}\right)} q_{k}+\sum_{k \in L_{\text {in }}\left(t_{l}\right)} q_{k} \leq 1$, we have $\frac{q_{j}}{1-q_{\text {in }}\left(t_{l}\right)}-1 \leq 0$. Then the function $\log \left(1-\sum_{k \in L_{i n}\left(t_{l}\right)} e^{\hat{q}_{k}}\right)$ is concave with respect to $\hat{q}$. With the same manipulation, we can prove that $\log \left(1-\sum_{k \in L_{\text {out }}\left(r_{l}\right)} e^{\hat{q}_{k}}\right)$ is concave with respect to $\hat{\boldsymbol{q}}$. Furthermore, $\log \left(\sum_{s: l \in L(s)} e^{\hat{x}_{s}}\right)$, $\log \left(\sum_{l \in L(v)} e^{\hat{q}_{l}}\right)$ are, respectively, convex with respect to $\hat{\boldsymbol{x}}, \hat{\boldsymbol{q}}$ due to the convexity of $\log$ of a sum of exponential of linear functions. Thus we proof the constraints set in (9) is convex. The proof is completed.

\section{APPENDIX B \\ PROOF OF THEOREM 2}

Proof: First, we show that $\boldsymbol{q}^{(t)}, \boldsymbol{p}^{(t)}$ are bounded with the subgradient algorithm (26) and (27). Denote $\boldsymbol{w}^{(t)}=$ $\left(\boldsymbol{q}^{(t)}, \boldsymbol{p}^{(t)}\right), \boldsymbol{W}^{*}(\boldsymbol{\lambda}, \boldsymbol{\mu})=\left(\boldsymbol{q}^{*}(\boldsymbol{\lambda}, \boldsymbol{\mu}), \boldsymbol{p}^{*}(\boldsymbol{\lambda}, \boldsymbol{\mu})\right)$ be the set of optimal solutions of (14) with a given $(\boldsymbol{\lambda}, \boldsymbol{\mu})$. We further define $d(\boldsymbol{w}, \boldsymbol{W})=\min _{\boldsymbol{w}^{\prime} \in \boldsymbol{W}}\left\|\boldsymbol{w}-\boldsymbol{w}^{\prime}\right\|$ and $N_{r}(\boldsymbol{W})=$ $\{\boldsymbol{w} \mid d(\boldsymbol{w}, \boldsymbol{W}(\boldsymbol{\mu})) \leq r\}$. We have the following results readily derived based on the convergence properties of subgradient algorithm [22]. Let $\left\{\boldsymbol{w}^{(t)}(\alpha)\right\}$ denote the sequence of vectors defined by the iterative procedure stated in (26) and (27) with $\alpha^{(t)}=\alpha, \forall t$. Then there exists a $K<\infty$ and a function $r(\alpha)$ such that $\lim _{\alpha \rightarrow 0^{+}} r(\alpha)=0$, and for all $\kappa>K$,

$$
\lim _{t \rightarrow \infty} d\left(\boldsymbol{w}^{(t)}, N_{r(\alpha)}\left(\boldsymbol{W}^{*}(\boldsymbol{\mu})\right)\right)=0 .
$$

Thus, $\boldsymbol{q}^{(t)}, \boldsymbol{p}^{(t)}$ are bounded. Next, we show the subgradient algorithm (17)-(18) with constant step size $\beta$ converges statistically to optimal value $\left(\boldsymbol{\lambda}^{*}, \boldsymbol{\mu}^{*}\right)$ of the dual function $D(\boldsymbol{\lambda}, \boldsymbol{\mu})$. Define the following Lyapunov function

$$
\mathcal{V}\left(\boldsymbol{\lambda}^{(t)}, \boldsymbol{\mu}^{(t)}\right)=\frac{1}{2 \beta} \sum_{l \in L}\left\{\left(\lambda_{l}^{(t)}-\lambda_{l}^{*}\right)^{2}+\left(\mu_{l}^{(t)}-\mu_{l}^{*}\right)^{2}\right\}
$$

By (17)-(18), we have Eq. (29), where the first inequality in Eq. (29) is due to $(\max [z, 0])^{2} \leq z^{2}$. 
(a)

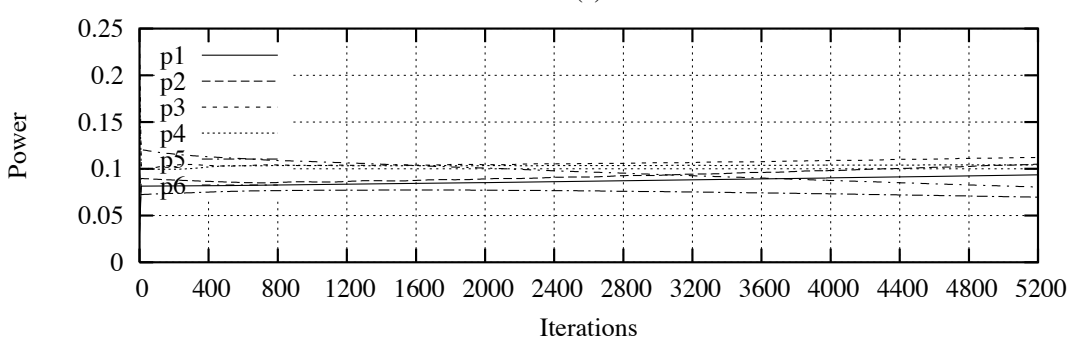

(b)

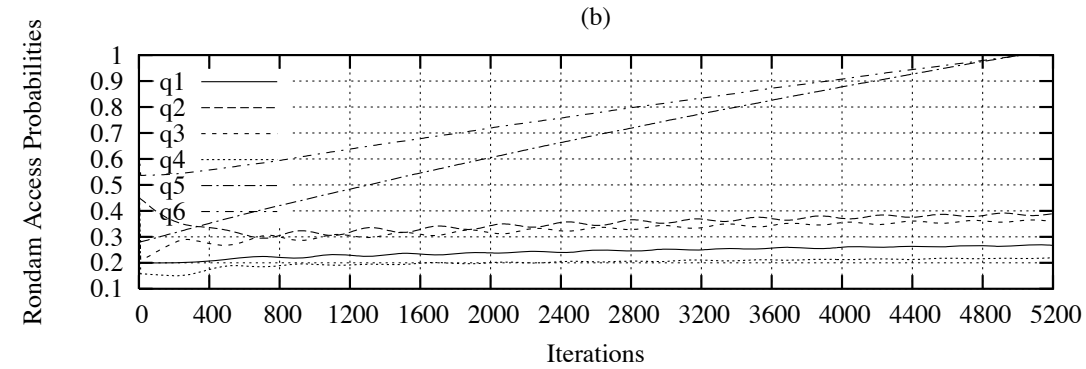

(c)

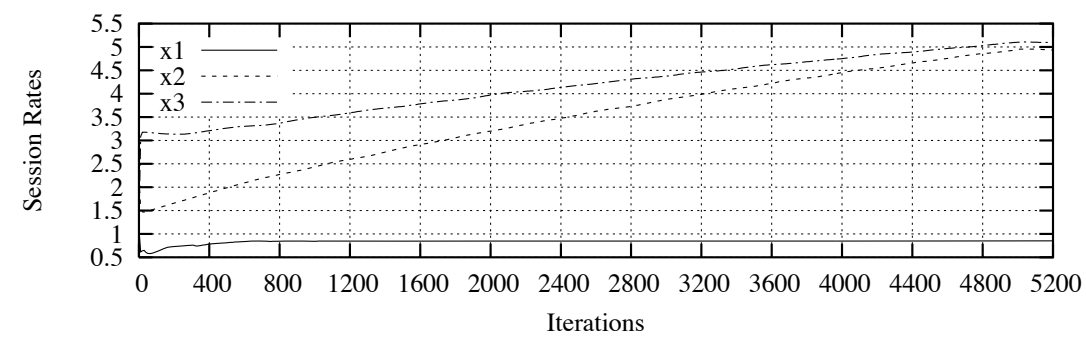

(d)

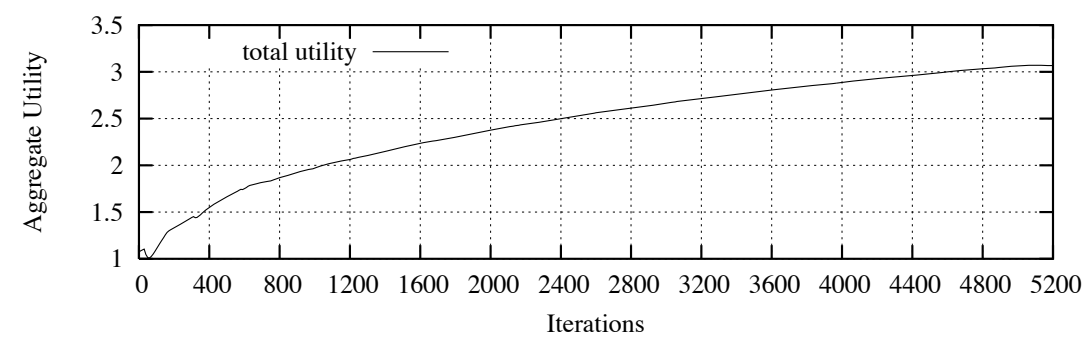

Fig. 10. Convergence dynamics when the distributed algorithm with interference-based communication model is used: (a) link power levels; (b) link access probabilities; (c) end-to-end rates; (d) aggregate utility.

Note that

$$
\begin{aligned}
& D\left(\boldsymbol{\lambda}^{(t)}, \boldsymbol{\mu}^{(t)}\right)-D\left(\boldsymbol{\lambda}^{*}, \boldsymbol{\mu}^{*}\right) \\
\geq & \sum_{l \in L} \hat{\mathcal{C}}_{l}\left(\lambda_{l}^{(t)}-\lambda_{l}^{*}\right)+\sum_{l \in L} \hat{\mathcal{D}}_{l}\left(\mu_{l}^{(t)}-\mu_{l}^{*}\right)
\end{aligned}
$$

Substituting into $(29)$, we obtain

$$
\begin{aligned}
& \mathcal{V}(\boldsymbol{\lambda}(t+1), \boldsymbol{\mu}(t+1)) \leq \mathcal{V}(\boldsymbol{\lambda}(t), \boldsymbol{\mu}(t)) \\
& -D(\boldsymbol{\lambda}(t), \boldsymbol{\mu}(t))+D\left(\boldsymbol{\lambda}^{*}, \boldsymbol{\mu}^{*}\right)+\frac{\beta M}{2}
\end{aligned}
$$

where $\quad \sum_{l \in L}\left(\log \frac{\sum_{s: l \in L(s)} x_{s}^{(t)}}{q_{l} \theta_{l} \log \left(1+y_{l}^{(t)}\right)}\right)^{2}$

$\sum_{l \in L}\left(\hat{y}_{l}^{(t)}-\log \frac{h_{t_{l} r_{l}} p_{l}^{(t)}}{\hat{\mathcal{I}}^{(t)}}\right)^{2} \leq Y$. Since $\hat{\boldsymbol{x}}^{(t)}, \hat{\boldsymbol{y}}^{(t)}$, $\hat{\boldsymbol{p}}^{(t)}, \hat{\boldsymbol{q}}^{(t)}$ are bounded, there exists $Y<+\infty$. Applying inequality (30) recursively, we have

$$
\begin{aligned}
& \mathcal{V}\left(\boldsymbol{\lambda}^{(t+1)}, \boldsymbol{\mu}^{(t+1)}\right) \leq \mathcal{V}\left(\boldsymbol{\lambda}^{(1)}, \boldsymbol{\mu}^{(1)}\right) \\
& -\sum_{\tau=1}^{t}\left(D\left(\boldsymbol{\lambda}^{(\tau)}, \boldsymbol{\mu}^{(\tau)}\right)-D\left(\boldsymbol{\lambda}^{*}, \boldsymbol{\mu}^{*}\right)\right)+\frac{\beta t Y}{2}
\end{aligned}
$$

From (31), we have

$$
\begin{aligned}
& \frac{\sum_{\tau=1}^{t}\left(-D\left(\boldsymbol{\lambda}^{*}, \boldsymbol{\mu}^{*}\right)+D\left(\boldsymbol{\lambda}^{(\tau)}, \boldsymbol{\mu}^{(\tau)}\right)\right)}{t} \\
\leq & \frac{\mathcal{V}\left(\boldsymbol{\lambda}^{(1)}, \boldsymbol{\mu}^{(1)}\right)}{t}+\frac{\beta Y}{2} .
\end{aligned}
$$

Since $D(\boldsymbol{\lambda}, \boldsymbol{\mu})$ is a concave function on $\boldsymbol{\lambda}, \boldsymbol{\mu}$, by Jenson's inequality, $\frac{1}{t} \sum_{\tau=1}^{t} D\left(\boldsymbol{\lambda}^{(\tau)}, \boldsymbol{\mu}^{(\tau)}\right) \leq D(\overline{\boldsymbol{\lambda}}(t), \overline{\boldsymbol{\mu}}(t))$, where $\overline{\boldsymbol{\lambda}}(t)=\frac{1}{t} \sum_{\tau=1}^{t} \boldsymbol{\lambda}^{(\tau)}, \overline{\boldsymbol{\mu}}(t)=\frac{1}{t} \sum_{\tau=1}^{t} \boldsymbol{\mu}^{(\tau)}$. Further taking 


$$
\begin{aligned}
& \mathcal{V}\left(\boldsymbol{\lambda}^{(t+1)}, \boldsymbol{\mu}^{(t+1)}\right) \\
\leq & \frac{1}{2 \beta} \sum_{l \in L}\left(\lambda_{l}^{(t)}+\beta \log \frac{\sum_{s: l \in L(s)} x_{s}^{(t)}}{q_{l} \theta_{l} \log \left(1+y_{l}^{(t)}\right)}-\lambda_{l}^{*}\right)^{2}+\frac{1}{2 \beta} \sum_{l \in L}\left(\mu_{l}^{(t)}+\beta\left(\hat{y}_{l}^{(t)}-\log \frac{h_{t_{l} r_{l}} p_{l}^{(t)}}{\hat{\mathcal{I}}^{(t)}}\right)-\mu_{l}^{*}\right)^{2} \\
= & \frac{1}{2 \beta} \sum_{l \in L}\left(\lambda_{l}(t)-\lambda_{l}^{*}\right)^{2}+\sum_{l \in L}\left\{\log \frac{\sum_{s: l \in L(s)} x_{s}^{(t)}}{q_{l} \theta_{l} \log \left(1+y_{l}^{(t)}\right)}\left(\lambda_{l}^{(t)}-\lambda_{l}^{*}\right)\right\}+\frac{\beta}{2} \sum_{l \in L}\left(\log \frac{\sum_{s: l \in L(s)} x_{s}^{(t)}}{q_{l} \theta_{l} \log \left(1+y_{l}^{(t)}\right)}\right)^{2} \\
& +\frac{\sum_{l \in L}\left(\mu_{l}^{(t)}-\mu_{l}^{*}\right)^{2}}{2 \beta}+\sum_{l \in L}\left\{\left(\hat{y}_{l}^{(t)}-\log \frac{h_{t_{l} r_{l}} p_{l}^{(t)}}{\hat{\mathcal{I}}^{(t)}}\right)\left(\mu_{l}^{(t)}-\mu_{l}^{*}\right)\right\}+\frac{\beta}{2} \sum_{l \in L}\left(\hat{y}_{l}^{(t)}-\log \frac{h_{t_{l} r_{l}} p_{l}^{(t)}}{\hat{\mathcal{I}}^{(t)}}\right)^{2}
\end{aligned}
$$

limits as $t \rightarrow \infty$, yields

$$
\lim \sup _{t \rightarrow \infty}\left(D\left(\overline{\boldsymbol{\lambda}}^{(t)}, \overline{\boldsymbol{\mu}}^{(t)}\right)-D\left(\boldsymbol{\lambda}^{*}, \boldsymbol{\mu}^{*}\right)\right) \leq \frac{\beta Y}{2} .
$$

Thus, according to the definition of statistical stability in [3], the subgradient algorithm (17)-(18) converges statistically to within $\frac{\beta Y}{2}$ of the optimal value $\left(\boldsymbol{\lambda}^{*}, \boldsymbol{\mu}^{*}\right)$. Then, given small enough positive constants $\alpha, \beta$ and large enough positive constant $\kappa$, the distributed JOCRP algorithm converges statistically to the global optimum of the JCRP problem (7). The proof is completed.

\section{REFERENCES}

[1] D. Bertsekas, Nonlinear Programming, 2nd ed., Athena scientific, 1999.

[2] L. Bui, A. Eryilmaz, R. Srikant, X. Wu, "Joint asynchronous congestion control and distributed scheduling for multi-hop wireless networks," in Proc. IEEE INFOCOM, Barcelona, Spain, April 2006.

[3] L. Chen, S. Low and J. Doyle, "Joint congestion control and media access control design for ad hoc wireless networks," in Proc. IEEE INFOCOM, Miami, FL, March 2005.

[4] L. Chen, S. Low, M. Chiang and J. Doyle, "Cross-layer congestion control, routing and scheduling design in ad hoc wireless networks," in Proc. IEEE INFOCOM, Barcelona, Spain, April 2006.

[5] M. Chiang, "Balancing transport and physical layers in wireless multihop networks: jointly optimal congestion control and power control," IEEE J. Select Areas Commun., vol. 23, no. 1, pp. 104-116, Jan. 2005.

[6] R. L. Cruz and A. V. Santhanam, "Optimal routing, link scheduling and power control in multi-hop wireless networks," in Proc. IEEE INFOCOM, San Francisco, April 2003.

[7] B. Johansson, P. Soldati, and M. Johansson, "Mathematical decomposition techniques for distributed cross-layer optimization of data networks," IEEE J. Select. Areas Commun., vol. 24, no. 8, pp. 15351547, Aug. 2006.

[8] F. P. Kelly, A. K. Maulloo and D. K. H. Tan, "Rate control for communication networks: Shadow prices, proportional fairness and stability," J. Operations Research Society, vol.49, no. 3, pp. 237-252, March 1998.

[9] M. Kodialam and T. Nandagopal, "Characterizing achievable rates in multi-hop wireless networks: The joint routing and scheduling problem," in Proc. ACM Mobicom, September 2003.

[10] J. W. Lee, M. Chiang, and R. A. Calderbank, "Utility-optimal randomaccess control," IEEE Trans. Wireless Commun., vol. 6, no. 7, pp. 27412751, July 2007.

[11] X. Lin and N. B. Shroff, "Joint rate control and scheduling in multihop wireless networks," in Proc. IEEE CDC, Paradise Island, Bahamas, Dec. 2004.

[12] X. Lin and N. Shroff, "The impact of imperfect scheduling on crosslayer rate control in multi-hop wireless networks," in Proc. IEEE INFOCOM, Miami, Florida, Mar. 2005.

[13] X. Lin, N. Shroff, and R. Srikant, "A tutorial on cross-layer optimization in wireless networks," IEEE J. Select. Areas Commun., vol. 24, no. 8, pp. 1452-1463, Aug. 2006.
[14] S. H. Low and D. E. Lapsley, "Optimization flow control, I: basic algorithm and convergence," IEEE/ACM Trans. Networking, vol. 7, no. 6, pp. 861-874, Dec. 1999.

[15] S. H. Low, "A duality model of TCP and queue management algorithms," IEEE/ACM Trans. Networking, vol. 11, no. 4, pp. 525-536, Aug. 2003.

[16] S. H. Low, F. Paganini and J. C. Doyle, "Internet congestion control," IEEE Control Syst. Mag., vol. 22, no. 1, pp.28-43, Feb. 2002.

[17] T. Moscibroda, R. Wattenhofer, and A. Zollinger, "The complexity of connectivity in wireless networks," in Proc. IEEE INFOCOM, Barcelona, Spain, April 2006.

[18] M. Neely, E. Modiano and C. Rohrs, "Dynamic power allocation and routing for time varying wireless networks," in Proc. IEEE INFOCOM, San Francisco, April 2003.

[19] D. P. Palomar, M. Chiang, "A tutorial on decomposition methods for network utility maximization," IEEE J. Select. Areas Commun., vol. 24, no. 8, pp. 1439-1451, Aug. 2006.

[20] J. Price and T. Javidi, "Distributed rate assignments for simultaneous interference and congestion control in CDMA-Based wireless networks," Submitted to IEEE Trans. Veh. Technol., 2007.

[21] G. Sharma, N. B. Shroff, and R. R. Mazumdar, "On the complexity of scheduling in wireless networks," in ACM Mobicom 2006 , Los Angeles, CA, Sept. 2006.

[22] N. Z. Shor, Minimization Methods for Non-Differentiable Functions. Springer-Verlag, 1985.

[23] X. Wang and K. Kar, "Cross-layer rate control for end-to-end proportional fairness in wireless networks with random access," in MobiHoc'05, Urbana-Champaign, Illinois, USA, May 2005.

[24] Q. Wu, Y. Xiong, Q. Zhang, Z. Guo, X. Xia, and Z. Li, "Joint routing and topology formation in multi-hop UWB networks," IEEE J. Select. Areas Commun., vol, 24, no. 4, pp. 843 - 849, April 2006.

[25] Y. Xue, B. Li, and K. Nahrstedt, "Optimal resource allocation in wireless ad hoc networks: a price-based approach," IEEE Trans. Mobile Computing, vol. 5, no. 4, pp. 347-364, April 2006.

[26] Y. Yi and S. Shakkottai, "Hop-by-hop congestion control over a wireless multi-hop network," in Proc. IEEE INFOCOM, Hong Kong, March 2004.

[27] J. Zhang, D. Zheng, and M. Chiang, "The impact of stochastic noisy feedback on distributed network utility maximization in multi-hop wireless networks," Submitted to IEEE Trans. Inform. Theory, February 2007.

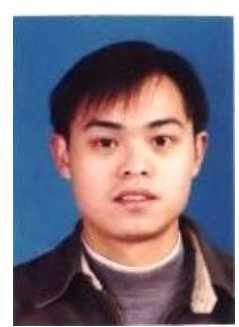

Chengnian Long (M'07) received the BS, MS, and $\mathrm{PhD}$ degrees from Yanshan University, China, in 1999, 2001, and 2004, respectively, all in control theory and engineering. He is an associate professor at Yanshan University. He visited Department of Computer Science and Engineering, Hongkong University of Science and Technology in 2006. Now he is a Killam postdoctoral fellow at Department of Electrical and Computer Engineering, University of Alberta. His current research interests are in the area of noncooperative behavior and incentive mechanism design in wireless multi-hop networks, energy-efficiency protocol design in wireless sensor networking, and pricing mechanism in Internet. 


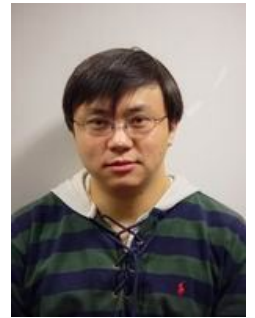

Bo Li (S'89-M'92-SM'99) received his B. Eng. (summa cum laude) and M. Eng. degrees in the Computer Science from Tsinghua University, Beijing in 1987 and 1989, respectively, and the Ph.D. degree in the Electrical and Computer Engineering from University of Massachusetts at Amherst in 1993. Between 1993 and 1996, he worked on high performance routers and ATM switches in IBM Networking System Division, Research Triangle Park, North Carolina. Since 1996, he has been with the Department of Computer Science, Hong Kong University of Science and Technology. Since 1999, he has also held an adjunct researcher position at the Microsoft Research Asia (MSRA), Beijing, China. His current research interests are on adaptive video multicast, peer-to-peer streaming, resource management in mobile wireless systems, across layer design in multihop wireless networks, content distribution and replication. He has published 100 journal papers and held several patents. $\mathrm{He}$ made original contributions on proxy placement problem in $\mathrm{CDN}$, capacity calculation in cellular networks, adaptive video multicast. Specifically the system Coolstreaming (Google entries over 400,000) attracted millions of download and was cited as the benchmark of the first large-scale Peer-to-Peer live video streaming system worldwide. He received the Oversea Outstanding Young Investigator Award from the National Natural Science Foundation of China (NSFC) in 2004. He serves as a Distinguished Lecturer for IEEE Communications Society (2006-2008).

$\mathrm{He}$ is the editor-in-chief for International Journal on Vehicular Technology. $\mathrm{He}$ has been on editorial board for IEEE Transactions on Wireless Communications, IEEE Transactions on Mobile Computing, IEEE Transactions on Vehicular Technology, IEEE Communications Survey and Tutorials, IEEE Journal of Selected Areas in Communications (J-SAC) -Wireless Communications Series, ACM/Kluwer Journal of Wireless Networks (WINET), ACM Mobile Computing and Communications Review (MC2R), Elsevier Ad Hoc Networks, SPIE/Kluwer Optical Networking Magazine (ONM), KICS/IEEE Journal of Communications and Networks (JCN). He served as a guest editor for IEEE Communications Magazine Special Issue on Active, Programmable, and Mobile Code Networking (April 2000), ACM Performance Evaluation Review Special Issue on Mobile Computing (December 2000), and SPIE/Kluwer Optical Networks Magazine Special Issue in Wavelength Routed Networks: Architecture, Protocols and Experiments (January/February 2002), IEEE J-SAC Special Issue on Protocols for Next Generation Optical WDM Networks (October 2000), IEEE J-SAC Special Issue on Recent Advances in Service-Overlay Network (January 2004), and IEEE J-SAC Special Issue on Quality of Service Delivery in Variable Topology Networks (September 2004), ACM/Kluwer Mobile Networks and Applications (MONET) Special Issue on Energy Constraints and Lifetime Performance in Wireless Sensor Networks (2nd December 2005), and ACM/Springer MONET Special Issue on Advances in Wireless Mesh Networks (to appear in 2008). In addition, $\mathrm{He}$ has been involved in organizing over 50 conferences, esp. IEEE Infocom since 1996. He was the Co-TPC Chair for IEEE Infocom 2004.

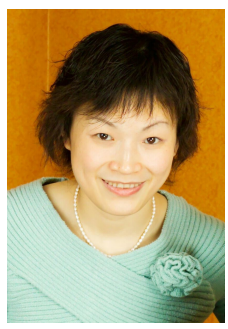

Qian Zhang (M'00-SM'04) received the BS, MS, and $\mathrm{PhD}$ degrees from Wuhan University, China, in 1994, 1996, and 1999, respectively, all in computer science. She joined the Hong Kong University of Science and Technology in September 2005 as an Associate Professor. Before that, she was at Microsoft Research Asia, Beijing, China, from July 1999, where she was the research manager of the Wireless and Networking Group. She has published about 200 refereed papers in international leading journals and key conferences in the areas of wireless/Internet multimedia networking, wireless communications and networking, and overlay networking. She is the inventor of about 30 pending patents. Her current research interests are in the areas of wireless communications, IP networking, multimedia, P2P overlay, and wireless security. She has also participated many activities in the IETF ROHC (Robust Header Compression) WG group for TCP/IP header compression.
Dr. Zhang is the associate editor for the IEEE Transactions on Wireless Communications, IEEE Transactions on Vehicular Technologies, IEEE Transactions on Multimedia, Elsevier Computer Networks and Elsevier Computer Communications. She has also served as guest editor for the IEEE Wireless Communications, IEEE Journal on Selected Areas in Communications, ACM/Springer Journal of Mobile Networks and Applications (MONET), and Computer Networks. Dr. Zhang received TR 100 (MIT Technology Review) world's top young innovator award in 2004, the Best Asia Pacific (AP) Young Researcher Award elected by the IEEE Communication Society in 2004, and the Best Paper Award by the Multimedia Technical Committee (MMTC) of the IEEE Communications Society and Best Paper Award in QShine 2006 and IEEE Globecom 2007. She received the Oversea Young Investigator Award from the National Natural Science Foundation of China (NSFC) in 2006. Dr. Zhang is Vice-Chair of the Multimedia Communication Technical Committee of the IEEE Communications Society.

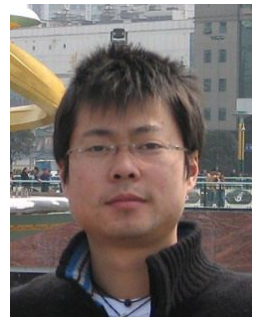

Bin Zhao received his B.S. and M.S. degrees in Applied Mathematics from Yanshan University, Qinhuangdao, China in 2002 and 2004, respectively. $\mathrm{He}$ is currently working toward the Ph.D. degree in the Department of Electrical Engineering, Yanshan University. His research interests include industrial wireless sensor networks, cognitive radio.

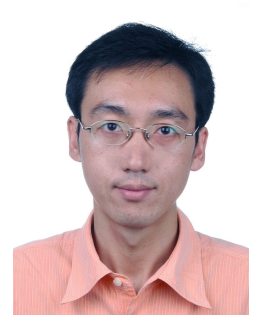

Bo Yang received his B.S. and M.S. degrees in Electrical Engineering from Yanshan University, Qinhuangdao, China in 2002 and 2004, respectively. $\mathrm{He}$ is currently working toward the Ph.D. degree in the Department of Manufacturing Engineering and Engineering Management, City University of Hong Kong, Kowloon, Hong Kong. During January to June 2007, Mr. Bo Yang visited the Department of Electrical and Computer Engineering, Polytechnic University, Brooklyn, NY, USA. His research interests include resource allocation in ad hoc networks and spectrum sharing in cognitive networks. He is a student member of IEEE.

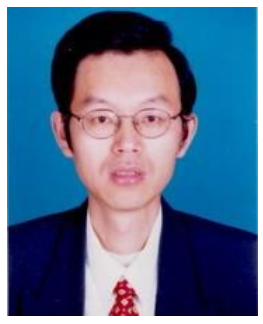

Xinping Guan received the B.S. degree in mathematics from Harbin Normal University, Harbin, China, and the M.S. degree in applied mathematics and the Ph.D. degree in electrical engineering, both from Harbin Institute of Technology, in 1986, 1991, and 1999, respectively. He is currently a Professor of the Department of Automation, Shanghai Jiaotong University, Shanghai, China. He is the (co)author of more than 100 papers in mathematical, technical journals, and conferences. As (a)an (co)-investigator, he has finished more than 19 projects supported by National Natural Science Foundation of China (NSFC), the National Education Committee Foundation of China, and other important foundations. His current research interests include wireless sensor networks, congestion control of networks, robust control and intelligent control for nonlinear systems. Dr. Guan is serving as an Associate Editor of IEEE Transaction on Systems, Man and Cybernetics (Part C), an Editor Board Member of International Journal of Systems, Control and Communications (IJSCC), a Reviewer of Mathematic Review of America, a Member of the Council of Chinese Artificial Intelligence Committee, and Vice-Chairman of Automation Society of Hebei Province, China. Dr. Guan is "Cheung Kong Scholars Programme" Special appointment professor, Ministry of Education, 2005, and National Science Fund for Distinguished Young Scholars of China, 2005. 